D.A. HERRON

KODAI MATH. J.

10 (1987), 9-19

\title{
THE HARNACK AND OTHER CONFORMALLY INVARIANT METRICS
}

\author{
By D. A. Herron*
}

1. Introduction. This paper investigates the Harnack metric $h_{D}$ in proper subdomains $D$ of euclidean space $\boldsymbol{R}^{n}$. By definition

$$
h_{D}\left(x_{1}, x_{2}\right)=\sup _{u}\left|\log \frac{u\left(x_{1}\right)}{u\left(x_{2}\right)}\right| \text {, }
$$

where the supremum is taken over all positive functions $u$ which are harmonic in $D$. Also useful is the quasihyperbolic metric [GP] given by

$$
k_{D}\left(x_{1}, x_{2}\right)=\inf _{\gamma} \int_{\gamma} \frac{|d z|}{d(z, \partial D)},
$$

where the infimum is taken over all locally rectifiable $\operatorname{arcs} \gamma$ joining $x_{1}, x_{2}$ in $D$.

First, we prove that $h_{D} \leqq n k_{D}$, and $n$ is the smallest constant for which this is true in all subdomains $D$ of $\boldsymbol{R}^{n}$. Next we show that if $G$ is a domain which has Green's functions $g(x, y)$ and if $D=G-E$, where $E$ is a relatively closed polar subset of $G$, then for all $x_{1}, x_{2} \in D$

$$
h_{D}\left(x_{1}, x_{2}\right)=\max \left\{h_{G}\left(x_{1}, x_{2}\right), \sup _{y \in E}\left|\log \frac{g\left(x_{1}, y\right)}{g\left(x_{2}, y\right)}\right|\right\} .
$$

Then we give a formula for the distance between two antipodal points on the circle of symmetry in an annulus. See $[\mathrm{K}]$, [L] and $[\mathrm{V}]$ for similar results.

Finally, we consider plane domains $D$ and we compare the Bergman metric $b_{D}$, the Carathéodory metric $c_{D}$, the Harnack metric $h_{D}$, and the Poincaré metric $p_{D}$. These metrics are all conformally invariant, and it is known that

$$
b_{D} \leqq p_{D} \text { and } c_{D} \leqq h_{D} \leqq p_{D} ;
$$

see $[A B],[B G],[K],[T]$. We give examples to show that these inequalities cannot be combined, and that for general plane domains the second set of inequalities is best possible. We also show that the strict inequality $h_{D}<p_{D}$ holds when $D$ has two or more isolated boundary points.

Received February 3, 1986

* This work was partially supported by grants from the U.S. National Science Foundation. 
Throughout this paper $D$ denotes a proper subdomain of euclidean space $\boldsymbol{R}^{n}$. The euclidean ball centered at $x$ of radius $r$ is denoted $B^{n}(x, r)$ and we use the abbreviations $B^{n}=B^{n}(0,1), B=B^{2}$. For $0<q<1$ fixed we let $A$ denote the annulus $A=\left\{z \in \boldsymbol{R}^{2}: \sqrt{q}<|z|<1 / \sqrt{q}\right\}$, and we work with the complete elliptic integrals and the Jacobian elliptic functions with modulus $k=k(q)$; see [BF]. Explicityly, $k=4 \sqrt{ } \bar{q} \prod_{1}^{\infty}\left(\frac{1+q^{2 n}}{1-q^{2 n}}\right)^{4}[\mathrm{~N}$, p. 289 , (30)].

By a metric we mean a pseudo-distance function. With each non-negative measurable function $\lambda$ defined in $D$ we associate a metric $\rho_{\lambda}$ by

$$
\rho_{\lambda}\left(x_{1}, x_{2}\right)=\inf _{\gamma} \int_{\gamma} \lambda(x)|d x|,
$$

where the infimum is taken over all locally rectifiable $\operatorname{arcs} \gamma$ joining $x_{1}, x_{2}$ in $D$. With each metric $\rho$ defined in $D$ we associate a non-negative function $\partial \rho$, the metric-density of $\rho$, by

$$
\partial \rho(x)=\limsup _{y \rightarrow x} \frac{\rho(x, y)}{|x-y|} .
$$

If is not difficult to show that when $\lambda$ is continuous

$$
\partial \rho_{\lambda}=\lambda \text {, }
$$

and that when $\partial \rho$ is continuous

$$
\rho \leqq \rho_{\partial \rho} .
$$

This work is part of the author's Ph.D. thesis which was written at the University of Michigan under the supervision of Professor Frederick W. Gehring, whom the author gratefully acknowledges.

2. The Harnack Metric. The Harnack metric, abbreviated H-metric, is defined in $D$ by

$$
h_{D}\left(x_{1}, x_{2}\right)=\sup _{u}\left|\log \frac{u\left(x_{1}\right)}{u\left(x_{2}\right)}\right|,
$$

where the supremum is taken over all functions $u$ in the class $\mathscr{H}^{+}(D)$ of positive functions harmonic in $D$; see $[\mathrm{K}],[\mathrm{L}],[\mathrm{T}]$.

Employing normal family arguments and Taylor's theorem, one can prove

THEOREM 1. For $x \in D \subset \boldsymbol{R}^{n}$,

$$
\partial h_{D}(x)=\sup _{u}|\nabla \log u(x)|,
$$

where the supremum is taken over all functions $u \in \mathscr{H}^{+}(D)$. 
We now derive a sharp upper bound for the $H$-metric. Notice that we have

$$
h_{D}\left(x_{1}, x_{2}\right) \leqq \log M
$$

if and only if we have the Harnack type inequality

$$
\frac{1}{M} \leqq \frac{u\left(x_{1}\right)}{u\left(x_{2}\right)} \leqq M \quad \text { for all } u \in \mathscr{H}^{+}(D) .
$$

The quasihyperbolic metric is defined in $D$ by

$$
k_{D}\left(x_{1}, x_{2}\right)=\rho_{\lambda}\left(x_{1}, x_{2}\right), \quad \lambda(x)=d(x, \partial D)^{-1},
$$

where $d(x, \partial D)$ is the euclidean distance from $x$ to $\partial D$. When $D$ is a halfspace this is the classical hyperbolic, or Poincaré, metric.

THEOREM 2. For $x_{1}, x_{2} \in D \subset \boldsymbol{R}^{n}$,

$$
h_{D}\left(x_{1}, x_{2}\right) \leqq n k_{D}\left(x_{1}, x_{2}\right),
$$

and $n$ is the smallest constant for which this is true in all subdomains $D$ of $\boldsymbol{R}^{n}$.

Proof. We first establish the infinitesimal version of our result,

$$
\frac{\left|\nabla u\left(x_{0}\right)\right|}{u\left(x_{0}\right)} \leqq \frac{n}{r} \quad \text { for all } u \in \mathscr{H}^{+}\left(B^{n}\left(x_{0}, r\right)\right) \text {. }
$$

Fix $u \in \mathscr{H}^{+}\left(B^{n}\left(x_{0}, r\right)\right)$. Then by Harnack's inequality [CH2]

$$
\left(\frac{r}{r+\rho}\right)^{n-2} \frac{r-\rho}{r+\rho} \leqq \frac{u(x)}{u\left(x_{0}\right)} \leqq\left(\frac{r}{r-\rho}\right)^{n-2} \frac{r+\rho}{r-\rho}
$$

for all $x, \rho$ with $\left|x-x_{0}\right| \leqq \rho<r$. Letting $t=\left|x-x_{0}\right| / r$ for $x \in B^{n}\left(x_{0}, r\right)$ we obtain

$$
1-n t+O\left(t^{2}\right) \leqq \frac{u(x)}{u\left(x_{0}\right)} \leqq 1+n t+O\left(t^{2}\right)
$$

as $t \rightarrow 0$, and hence

$$
-\frac{n}{r}+O\left(\left|x-x_{0}\right|\right) \leqq \frac{u(x)-u\left(x_{0}\right)}{u\left(x_{0}\right)\left|x-x_{0}\right|} \leqq \frac{n}{r}+O\left(\left|x-x_{0}\right|\right)
$$

as $\left|x-x_{0}\right| \rightarrow 0$, which establishes (4).

Now Theorem 1 and (4) yield

whence by (1) and (2)

$$
\partial h_{D} \leqq n \partial k_{D},
$$

$$
h_{D} \leqq \rho_{\partial h_{D}} \leqq \rho_{n \partial k_{D}}=n k_{D} .
$$

To see that $n$ is the smallest such constant we compute the distances between the origin and a nearby point in $B^{n}$. We have 


$$
k_{B^{n}}(0, x)=\int_{[0, x]} \frac{|d z|}{1-|z|}=\log \frac{1}{1-|x|},
$$

and (from $[\mathrm{K}]$ or $[\mathrm{L}]$ )

$$
h_{B^{n}}(0, x)=\log \frac{1+|x|}{(1-|x|)^{n-1}} .
$$

Thus

$$
h_{B^{n}}(0, x)-n k_{B^{n}}(0, x)=\log \left(1-|x|^{2}\right),
$$

and given $c<n$ one can find an $x \in B^{n}$ such that

$$
h_{B^{n}}(0, x)>c k_{B^{n}}(0, x) \text {. }
$$

Leutwiler has shown [L, Lemma 5.4] that a lower bound for the $H$-metric is given by

$$
h_{D}\left(x_{1}, x_{2}\right) \geqq(n-2)\left|\log \frac{d\left(x_{1}, \partial D\right)}{d\left(x_{2}, \partial D\right)}\right| .
$$

If $D$ has isolated boundary points, then Theorem 3 below also gives lower bounds. Moreover, since the $H$-metric is known in balls and half-spaces, Theorem 3 gives a large class of domains for which we can compute the $H$-metric.

$A$ set $E \subset \boldsymbol{R}^{n}$ is polar if there exist an open set $U \supset E$ and a subharmonic function $u$ defined on $U$ with $\left.u\right|_{E} \equiv-\infty$.

THEOREM 3. Let $G \subset \boldsymbol{R}^{n}$ be a domain which has a Green's function $g(x, y)$. Let $E \subset G$ be a relatively closed polar set, and let $D=G-E$. Then

$$
h_{D}\left(x_{1}, x_{2}\right)=\max \left\{h_{G}\left(x_{1}, x_{2}\right), \sup _{y \in E}\left|\log \frac{g\left(x_{1}, y\right)}{g\left(x_{2}, y\right)}\right|\right\}
$$

for all $x_{1}, x_{2} \in D$.

Proof. Fix $x_{1}, x_{2} \in D$. Since $D \subset G$,

$$
h_{D}\left(x_{1}, x_{2}\right) \geqq h_{G}\left(x_{1}, x_{2}\right) \text {. }
$$

If $y \in E$, then $g(\cdot, y) \in \mathscr{H}^{+}(D)$, so

$$
h_{D}\left(x_{1}, x_{2}\right) \geqq\left|\log \frac{g\left(x_{1}, y\right)}{g\left(x_{2}, y\right)}\right| \text {. }
$$

Thus

$$
h_{D}\left(x_{1}, x_{2}\right) \geqq \log M,
$$

where $\log M$ denotes the right-hand side of (5).

For the reverse inequality, fix $u \in \mathscr{H}^{+}(D)$. Then $u$ has a unique superharmonic extension to $G$, and there exists a unique measure $\mu$ on $G$ such that

$$
u(x)=v(x)+\int_{G} g(x, y) d \mu(y),
$$


where $v$ is the greatest harmonic minorant of $u$ in $G$; see [He]. Now since $u$ is harmonic in $D, \mu$ is supported in $E$. Thus, using the definition of $M$ we obtain

$$
u\left(x_{1}\right)=v\left(x_{1}\right)+\int_{E} g\left(x_{1}, y\right) d \mu(y) \leqq M v\left(x_{2}\right)+M \int_{E} g\left(x_{2}, y\right) d \mu(y)=M u\left(x_{2}\right) .
$$

Hence by symmetry

$$
\frac{1}{M} \leqq \frac{u\left(x_{1}\right)}{u\left(x_{2}\right)} \leqq M
$$

and thus

$$
h_{D}\left(x_{1}, x_{2}\right) \leqq \log M
$$

Next, suppose $D$ has a Poisson kernel, that is, suppose there exists a positive function $P(\xi, x)$, defined for $\xi \in \partial D, x \in D$ such that

$$
u(x)=\int_{\partial D} P(\xi, x) u(\xi) d \sigma(\xi)
$$

for all $u$ harmonic in $D$ and continuous in $\bar{D}$, where $\sigma$ is surface area measure on $\partial D$. We remark that for each fixed $\xi \in \partial D, P(\xi, x)$ is a harmonic function of $x \in D$.

Fix $x, y \in D$ and consider

$$
f(\xi)=\frac{P(\xi, x)}{P(\xi, y)}, \quad \xi \in \partial D
$$

Choose $\xi_{1}, \xi_{2} \in \partial D$ so that

$$
m=f\left(\xi_{1}\right)=\min _{\partial D} f, \quad M=f\left(\xi_{2}\right)=\max _{\partial D} f .
$$

Suppose $u$ is positive, harmonic in $D$, and continuous in $\bar{D}$. Then

$$
m u(y)=m \int_{\partial D} P(\xi, y) u(\xi) d \sigma(\xi) \leqq \int_{\partial D} P(\xi, x) u(\xi) d \sigma(\xi)=u(x) \leqq M u(y) .
$$

As there is equality in the first, second inequality for

$$
u_{1}(z)=\frac{P\left(\xi_{1}, z\right)}{P\left(\xi_{1}, y\right)}, \quad u_{2}(z)=\frac{P\left(\xi_{2}, z\right)}{P\left(\xi_{2}, y\right)},
$$

respectively, we conclude 
THEOREM 4.

$$
h_{D}(x, y)=\log \max \left\{M, \frac{1}{m}\right\} .
$$

This is the method we use to study the $H$-metric in an annulus.

THEOREM 5. The Harnack distance between the points $1,-1 \in A$ is given by

$$
h_{A}(1,-1)=\log \frac{1+k}{1-k} \text {. }
$$

Proof. By Theorem 4 and symmetry

$$
h_{D}\left(1, e^{i \theta}\right)=\log M,
$$

where $M$ is the maximum value of

$$
f(\zeta)=\frac{P(\zeta, 1)}{P\left(\zeta, e^{i \theta}\right)} \quad(\zeta \in \partial A)
$$

and $P(\zeta, z)$ is the Poisson kernel for $A$.

By [CH1] the Green's function for $A$ with a pole at the point 1 is

$$
g(\zeta, 1)=-\operatorname{Re} \log \left(\sqrt{k} i \operatorname{sn}\left(\frac{K}{\pi i} \log \zeta\right)\right),
$$

and hence

$$
P(\zeta, 1)=\frac{\mp K}{2 \pi^{2}|\zeta|} \operatorname{Im}\left(\frac{d n u}{\operatorname{tn} u}\right)
$$

where $\zeta=q^{ \pm 1 / 2} e^{\imath \phi}, u=(K / \pi) \phi \pm\left(K^{\prime} / 2\right) i$. Thus

$$
f(\zeta)=\frac{\operatorname{Im}\left(\frac{\operatorname{dn} u}{\operatorname{tn} u}\right)}{\operatorname{Im}\left(\frac{\operatorname{dn} v}{\operatorname{tn} v}\right)},
$$

where $\zeta=q^{ \pm 1 / 2} e^{2 \phi}, u=(K / \pi) \phi \pm\left(K^{\prime} / 2\right) i, v=(K / \pi)(\phi-\theta) \pm\left(K^{\prime} / 2\right) i$. From [BF]

whence

$$
d n(u-K)=k^{\prime} / d n u, \quad \operatorname{tn}(u-K)=-1 / k^{\prime} \operatorname{tn} u,
$$

$$
f(\zeta)=\frac{\operatorname{Im}\left(\frac{\operatorname{dn} u}{\operatorname{tn} u}\right)}{-k^{\prime 2} \operatorname{Im}\left(\frac{\operatorname{tn} u}{\operatorname{dn} u}\right)}=\frac{1}{1-k^{2}}\left|\frac{d n u}{\operatorname{tn} u}\right|^{2}
$$

and the result follows as soon as we establish

$$
\max _{x \in R}\left|\frac{d n\left(x+\frac{K^{\prime}}{2} i\right)}{\operatorname{tn}\left(x+\frac{K^{\prime}}{2} i\right)}\right|=1+k .
$$


Using symmetry and standard mapping properties of $w=s n z$ it is not difficult to show that for $x \in \boldsymbol{R}$

$$
\left|\operatorname{sn}\left(x+\frac{K^{\prime}}{2} i\right)\right|=1 / \sqrt{k} .
$$

Then using the definitions of $c n z, d n z$ and mapping properties of $w=\left(1-z^{2}\right)^{1 / 2}$ we find that for $x \in \boldsymbol{R}$

$$
\left|d n\left(x+\frac{K^{\prime}}{2} i\right)\right|=\sqrt{k}\left|\operatorname{cn}\left(x+\frac{K^{\prime}}{2} i\right)\right| \leqq \sqrt{k}\left|\operatorname{cn}\left(\frac{K^{\prime}}{2} i\right)\right|=(1+k)^{1 / 2}
$$

Thus

$$
\left|\frac{d n}{t n}\left(x+\frac{K^{\prime}}{2} i\right)\right|=k\left|c n\left(x+\frac{K^{\prime}}{2} i\right)\right|^{2} \leqq 1+k
$$

as desired.

3. Other Metrics. We now restrict ourselves to proper subdomains $D$ of the complex plane $\boldsymbol{C}=\boldsymbol{R}^{2}$, where the Harnack metric is conformally invariant. That is, if $\phi: D \rightarrow D^{\prime}$ is a conformal mapping of $D$ onto $D^{\prime}$, then

$$
h_{D^{\prime}}\left(\phi\left(z_{1}\right), \phi\left(z_{2}\right)\right)=h_{D}\left(z_{1}, z_{2}\right)
$$

for all $z_{1}, z_{2} \in D$. We examine three other conformally invariant metrics: the Bergman, Carathéodory, and Poincaré metrics; and we compare all four metrics. Each metric arises as a solution to an extremal problem and relations among the metrics give information relating these extremal problems.

In the unit disk $B$ there is essentially one conformally invariant metric:

$$
\rho\left(z_{1}, z_{2}\right)=\log \frac{1+r}{1-r}, \quad r=\left|\frac{z_{1}-z_{2}}{1-\bar{z}_{2} z_{1}}\right| .
$$

The Carathéodory metric $c_{D}\left(z_{1}, z_{2}\right)$ and the Poincaré, or hyperbolic, metric $p_{D}\left(z_{1}, z_{2}\right)$ are defined in $D$ by

$$
\sup _{f} \rho\left(f\left(z_{1}\right), f\left(z_{2}\right)\right)
$$

where the suprema are taken over all functions $f$ in the classes $\mathscr{B}$ and $\mathscr{M}$ respectively. Here $\mathscr{M}$ is the class of all multiple-valued analytic functions $f: D \rightarrow B$ and $\mathscr{B}$ is the subclass of $\mathscr{M}$ of single-valued functions. Thus $\mathscr{M}$ consists of analytic continuations $f$, along curves in $D$, of single-valued analytic functions $f_{0}: B^{2}\left(z_{0}, r\right) \subset D \rightarrow B$, with each value of $f(z)$ in $B$ for all $z$ in $D$.

We have given a global definition for the Poincaré metric. In fact

where

$$
p_{D}=\rho_{\lambda_{D}}, \quad \lambda_{D}=\partial p_{D},
$$

$$
d s^{2}=\lambda_{D}(z)^{2}|d z|^{2}
$$


defines a Riemannian metric in $D$ with constant Gaussian curvature -1 . The Bergman metric is defined in $D$ by

$$
b_{D}\left(z_{1}, z_{2}\right)=\rho_{\kappa_{D}}\left(z_{1}, z_{2}\right), \quad \kappa_{D}(z)=2\left(\pi \widetilde{K}_{D}(z, \bar{z})\right)^{1 / 2},
$$

where $\tilde{K}_{D}$ is the reduced Bergman reproducing kernel for the Hilbert space

$$
\left\{f^{\prime}: f \text { analytic in } D, \iint_{D}\left|f^{\prime}(z)\right|^{2} d x d y<+\infty\right\}
$$

see $[B G]$, $[B]$.

3.1. Relations between metric-densities. Using (1), an extremal property of $\widetilde{K}_{D}$, Theorem 1 and its proof, and Schwarz' lemma we find that the metricdensities $\partial b_{D}, \partial c_{D}, \partial h_{D}, \partial p_{D}$ are each given by

$$
2 \sup _{f}\left|f^{\prime}(z)\right|,
$$

where the suprema are taken over all functions $f$ in $\mathscr{D}, \mathscr{B}, \mathscr{N}, \mathscr{M}$ respectively. Here

$$
\mathscr{D}=\left\{f \text { analytic in } D: \iint_{D}\left|f^{\prime}(z)\right|^{2} d x d y \leqq \pi\right\}
$$

and

$$
\mathscr{H}=\left\{f \in \mathscr{M}: \operatorname{Re}\left(\frac{1+f}{1-f}\right) \text { is single-valued }\right\} .
$$

From (6), $[\mathrm{AB}],[\mathrm{BG}]$, and the inclusions $\mathscr{B} \subset \mathscr{H} \subset \mathscr{M}$ we obtain

$$
\partial b_{D} \leqq \partial c_{D} \leqq \partial h_{D} \leqq \partial p_{D},
$$

and this is valid throughout each domain $D \subset C$.

At each point $e^{i \theta}$ in $A$ we have

$$
\left\{\begin{array}{l}
\partial b_{A}=\frac{2}{\pi}\left(K^{2}-K E\right)^{1 / 2} \\
\partial c_{A}=\frac{2}{\pi} K k \\
\partial p_{A}=\frac{K}{K^{\prime}}
\end{array}\right.
$$

see $[\mathrm{BG}],[\mathrm{Ha}],[\mathrm{Si}]$. From $[\mathrm{BF}]$ it follows that

$$
\partial b_{A}<\partial c_{A}<\partial p_{A}
$$

at each $e^{i \theta}$ in $A$. In fact for a non-simply, finitely connected domain $D$ with no degenerate boundary components we have

$$
\partial b_{D}<\partial c_{D}<\partial p_{D}
$$


see $[\mathrm{Sa} 1],[\mathrm{Sa} 2],[\mathrm{A}]$.

3.2. Relations between metrics. In each simply connected domain $D \subset C$

$$
b_{D}=c_{D}=h_{D}=p_{D} .
$$

For a general subdomain $D$ of $C$ we see from (7), [Sa3] that

$$
b_{D} \leqq p_{D}
$$

with equality if and only if $D$ is simply connected or $p_{D}=0$; and from $[\mathrm{K}]$, [T] we have

$$
c_{D} \leqq h_{D} \leqq p_{D}
$$

From (7) we expect that, locally,

$$
b_{D} \leqq c_{D} \leqq h_{D} \leqq p_{D} .
$$

However, we shall see that such inequalities are not, in general, valid globally. That is, (9) and (10) cannot be combined.

First we consider the punctured disk $D=B-\{0\}$. It is easy to see that

By Theorem 3

$$
b_{D}=c_{D}=p_{B} .
$$

$$
h_{D}\left(z_{1}, z_{2}\right)=\max \left\{p_{D}\left(\left|z_{1}\right|,\left|z_{2}\right|\right), p_{B}\left(z_{1}, z_{2}\right)\right\} .
$$

Thus on concentric circles

while on radial segments

$$
c_{D}=h_{D}<p_{D},
$$

$$
c_{D}<h_{D}=p_{D} .
$$

Therefore (10) cannot, in general, be improved.

THEOREM 6. Suppose $D$ is a domain with (at least) two isolated boundary points. Then

$$
h_{D}<p_{D}
$$

Proof. First, suppose $G \subset C$ is a domain which has a Green's function $g(z, \zeta)$. Fix $\zeta_{0} \in G$ and set $G_{0}=G-\left\{\zeta_{0}\right\}$. Then it is not hard to show that

$$
\left|\log \frac{g\left(z_{1}, \zeta_{0}\right)}{g\left(z_{2}, \zeta_{0}\right)}\right| \leqq p_{G_{0}}\left(z_{1}, z_{2}\right)
$$

for all $z_{1}, z_{2} \in G_{0}$.

To establish (11) we may assume that $D$ has a Green's function $g(z, \zeta)$. Choose isolated points $\zeta_{1}, \zeta_{2} \in \partial D$ and let 


$$
G=D \cup\left\{\zeta_{1}, \zeta_{2}\right\}, \quad G_{j}=G-\left\{\zeta_{j}\right\} \quad(j=1,2) .
$$

Then by Theorem 3 and the above we obtain

for all $z_{1}, z_{2} \in D$.

$$
\begin{aligned}
h_{D}\left(z_{1}, z_{2}\right) & =\max \left\{\left|\log \frac{g\left(z_{1}, \zeta_{1}\right)}{g\left(z_{2}, \zeta_{1}\right)}\right|,\left|\log \frac{g\left(z_{1}, \zeta_{2}\right)}{g\left(z_{2}, \zeta_{2}\right)}\right|, h_{G}\left(z_{1}, z_{2}\right)\right\} \\
& \leqq \max \left\{p_{G_{1}}\left(z_{1}, z_{2}\right), p_{G_{2}}\left(z_{1}, z_{2}\right), p_{G}\left(z_{1}, z_{2}\right)\right\} \\
& <p_{D}\left(z_{1}, z_{2}\right)
\end{aligned}
$$

Next we consider the annulus $A$. We remark that for $0 \leqq \theta \leqq \pi$ we have

$$
\left\{\begin{array}{l}
b_{A}\left(1, e^{i \theta}\right)=\frac{2 \theta}{\pi}\left(K^{2}-K E\right)^{1 / 2} \\
c_{A}\left(1, e^{i \theta}\right)=\log \frac{1+k \operatorname{sn}\left(\frac{\theta}{\pi}\right) K}{1-k \operatorname{sn}\left(\frac{\theta}{\pi}\right) K} \\
p_{A}\left(1, e^{i \theta}\right)=\theta \frac{K}{K^{\prime}} .
\end{array}\right.
$$

The first and last of these follow from (8) and the fact that the circle $\{z:|z|=1\}$ is a geodesic for any conformally invariant metric in $A$. The middle identity is contained in [Si], and it and Theorem 5 imply

$$
c_{A}(1,-1)=h_{A}(1,-1) .
$$

Finally, for the special case $q=e^{-\pi}$ we have

$$
k=1 / \sqrt{2}, \quad K=1.85 \cdots, \quad E=1.35 \cdots,
$$

whence by (12) and [BF]

while

$$
b_{A}(1, i)=0.96 \cdots<c_{A}(1, i)=1.21 \cdots
$$

$$
b_{A}(1,-1)=1.93 \cdots>c_{A}(1,-1)=1.76 \cdots .
$$

This shows that (9) and (10) cannot be combined, and that there is no simple relationship between either the Bergman and Carathéodory metrics or between the Bergman and Harnack metrics.

\section{REFERENCES}

[A] L. V. Ahlfors, Bounded analytic functions, Duke Math. J., 14 (1947), 1-11.

[AB] L. V. AhlFors AND A. BEURling, Conformal invariants and function-theoretic null-sets, Acta. Math., 83 (1950), 101-129.

[B] S. Bergman, The kernel function and conformal mapping, Amer. Math. Soc. 
Survey 5, New York 1950.

[BF] P.F. BYRD AND M.D. FRIEdman, Handbook of Elliptic Integrals for Engineers and Scientists, Springer-Verlag, New York 1971.

[BG] A.F. BEARDon AND F.W. GEHRING, Schwarzian derivatives, the Poincaré metric and the kernel function, Comment. Math. Helv., 55 (1980), 50-64.

[CH1] R. Courant and D. Hilbert, Methods of Mathematical Physics, Vol. 1, Interscience Publishers, New York, 1953.

[CH2] R. Courant and D. Hilbert, Methods of Mathematical Physics, Vol. 2, Interscience Publishers, New York, 1953.

[GP] F. W. Gehring and B.P. Palka, Quasiconformally homogeneous domains, J. d'Anal. Math., 30 (1976), 172-199.

[Ha] E. R. HANSEN, A Table of Series and Products, Prentice Hall, Englewood Cliffs, New Jersey 1975.

[He] L. L. Helms, Introduction to Potential Theory, John Wiley and Sons, New York, 1969.

[K] J. KöHN, Die Harnacksche Metrik in der Theorie der harmonischen Funktionen, Math. Zeitschr., 91 (1966), 50-64.

[L] H. Leutwiler, On a distance invariant under Mobius transformations in $\boldsymbol{R}^{n}$, Ann. Acad. Sci. Fenn. Ser. AI Math., to appear.

[N] Z. Nehari, Conformal Mapping, Dover Publications, Inc., New York, 1975.

[Sa1] M. SAKAI, On constants in extremal problems of analytic functions, Kōdai Math. Sem. Rep., 21 (1969), 223-225.

[Sa2] M. SAKAI, Correction to "On constants in extremal problems of analytic functions", Kōdai Math. Sem. Rep., 22 (1970), 128.

[Sa3] M. SAKAI, The sub-mean-value property of subharmonic functions and its application to the estimation of the Gaussian curvature of the span metric, Hiroshima Math. J., 9 (1979), 555-593.

[Si] R. R. Simha, The Carathéodory metric of the annulus, Proc. Amer. Math. Soc., 50 (1975), 162-166.

[T] H. TANAKA, On Harnack's pseudo-distance, Hokkaido Math. J., 6 (1977), 302-305.

[V] M. VuoRinen, On the Harnack constant and the boundary behavior of Harnack functions, Ann. Acad. Sci. Fenn. Ser. AI Math., 7 (1982), 259-277.

Department of Mathematical Sciences

UNIVERSITY OF CinCINNATI

Cincinnati, Ohio 45221 